\title{
28 Resarch Square on \\ Efficacy and safety of a ketogenic diet in children with drug-resistant epilepsy
}

\section{Juan Wang}

Chongqing Medical University Affiliated Children's Hospital

\section{Li Jiang ( $\sim$ lijiangcmu@126.com )}

Chongqing Medical University Affiliated Children's Hospital https://orcid.org/0000-0002-3010-6039

Yi Guo

Chongqing Medical University Affiliated Children's Hospital

\section{Min Cheng}

Chongqing Medical University Affiliated Children's Hospital

\section{Research}

Keywords: drug-resistant epilepsy, efficacy, ketogenic diet, paediatric, electroencephalograph

Posted Date: October 2nd, 2020

DOI: https://doi.org/10.21203/rs.3.rs-84927/v1

License: (c) (i) This work is licensed under a Creative Commons Attribution 4.0 International License. Read Full License 


\section{Abstract}

Background: To prospectively evaluate the efficacy and safety of a ketogenic diet (KD) in managing seizures in children with drug-resistant epilepsy and identify factors influencing its efficacy in a Chinese cohort.

Methods: Ninety-two pediatric patients (55 males, 37 females), aged 3 months to 10 years $(29.26 \pm 24.18$ months), with drug-resistant epilepsy who followed a KD for 1-12 months were included. The efficacy of the KD was evaluated by assessing the seizure frequency, as reported by parents/caregivers. The effects of demographic and clinical variables on patients' duration on the KD were analyzed.

Results: At the 1-, 3-, 6-, and 12-month follow-ups, $78.26 \%, 75 \%, 80 \%$, and $93.10 \%$ of patients were responsive to KD therapy, respectively. Five non-responders at 3 months became responsive by 6 months. Age, disease duration, seizure type, etiology, anti-epileptic drug (AED) type, and KD ratio did not affect treatment efficacy. After the KD, changes in AEDs and electroencephalography and imaging findings were statistically significant $(p<0.05)$. Only $4.34 \%$ patients experienced complications requiring medical intervention.

Conclusions: KD is an effective and well-tolerated therapy for children with drug-resistant epilepsy. The KD adherence rate largely depends on its efficacy. Genetic information relating to the diet response and synergistic effect between KD and AEDs requires further study.

Clinical trial has registered at Chinese clinical trial registry: http://www.chictr.org.cn

Registration numbers: ChiCTR2000031394. Date: (30/03/2020)

Availability of data and material: http://www.medresman.org.cn/uc/index.aspx

Code availability (software application or custom code): ziyan030815, wangjuan030815

\section{Background}

As a common neurological disorder, epilepsy affects approximately 68 million people worldwide ${ }^{[1]}$. In China, the prevalence is approximately $0.33-0.68 \%[2,3]$. Successful treatment of epilepsy in children remains a challenge; $25 \%$ of children with epilepsy experience refractory seizures ${ }^{[4,5]}$. The ketogenic diet (KD) has been used to manage seizures in children with epilepsy worldwide ${ }^{[6]}$. Randomized controlled trials have demonstrated that the KD reduces $50 \%$ of seizures in $50 \%$ of children with drug-resistant epilepsy ${ }^{[7]}$, but the factors affecting the efficacy of the KD remain unclear ${ }^{[8]}$. In this study, we aimed to evaluate the efficacy and side effects of KD therapy, as well as the changes in the electroencephalography (EEG) and magnetic resonance imaging (MRI) findings, in pediatric Chinese patients with refractory epilepsy. Finally, we sought to identify the factors influencing the efficacy of KD. 


\section{Material And Methods}

\subsection{Participants}

The participants comprised 92 children with a previously confirmed diagnosis of refractory epilepsy who were recruited from the Department of Neurology of the Children's Hospital of Chongqing Medical University in China between November 1, 2014, to October 1, 2018. Participants were recruited after physicians had recommended the Ketogenic diet. None of the patients had eceived KD treatment before and their physicians determined them to be suitable for KD treatment. Patient demographics were collected, including age, seizure type, etiology, and epileptic syndromes. Epilepsy and seizure types were classified using the recommendations of the International League Against Epilepsy $2010^{[9]}$. All patients underwent EEG, MRI, and metabolic assessment (urinary organic acid chromatography and blood amino acid chromatography).

\subsection{Implementation and clinical evaluation}

We received approval from the Institutional Review Board of the Children's Hospital of Chongqing Medical University. The participants' parents provided consent for participation in our study and were informed of the risks/benefits of study participation.

A non-fasting gradual initiation protocol of 2:1 KD therapy was initiated in the hospital under the guidance of a dietician.

Blood beta-hydroxybutyrate measurements obtained from fingertip whole blood samples were used to evaluate patients' ketone status. Ketone levels were measured using the FreeStyle Optium Neo Blood Glucose and Ketone Monitoring System (Abbott Diabetes Care Inc., USA). We monitored blood ketone levels once every $6 \mathrm{~h}$ when the patients were hospitalized. KD ratios varied from 1:1 to 4.5:1, depending on individual patients' blood ketone levels and the frequency of convulsions. Blood ketone levels reached target levels within 2-9 days with a mean of 4.5 days for all patients. The total hospitalization period was $7.20 \pm 1.56$ days (5-14 days). When ketone concentrations stabilized between 2 and $5 \mathrm{mmol} / \mathrm{L}[6,10]$ and there were no side effects, the child was discharged and followed-up by telephone calls and clinic visits. After discharge, ketones were monitored once daily for the first month and weekly thereafter.

Parents reported the blood ketone level, glucose level, seizure frequency, and any adverse events. At clinic visits, the KD ratio was adjusted depending on blood ketone levels and the degree of seizure control. The caloric intake was adjusted to maintain ideal body weight and height, based on the patient's height and weight gain or loss.

When the KD therapy elicited a positive response, we helped the patients to gradually reduce their AEDs, one at a time. Parents were asked about the effects of each AED, and if an AED led to significantly worse seizures, we considered reducing that drug dose first. We then reduced the drugs that had little to no effect. Finally, we eliminated the drugs that were initially effective, but were unable to maintain the effect.

\subsection{Follow-up analyses}


Patients' age, sex, seizure frequency, anticonvulsant use, etiology, KD ratio, blood, serum biochemistry, abdominal ultrasonography, EEG, MRI, and adverse effects were evaluated at baseline and at follow-ups during therapy. The caregivers were asked to maintain seizure, diet, and blood ketone and glucose monitoring notes, and mention any side effects or other reasons for stopping the diet to the physicians. After routine examination, some candidates did not follow the KD because they opted to undergo surgery or another treatment or their parents requested to withdraw. Some patients experienced allergic reactions, or refused food when introduced to the KD. For such reasons, 23 patients were not included the study.

\subsection{Criteria for efficacy evaluation}

Changes in seizure frequency were evaluated using daily seizure calendars recorded by caregivers. The seizure frequency at follow-ups was compared to the baseline frequency. The efficacy was evaluated as follows: (1) Seizure free: seizures disappeared; (2) Improvement: $\geq 50 \%$ reduction: seizure frequency decreased by $\geq 50 \%$ but did not disappear; (3) No improvement: $<50 \%$ reduction: seizure frequency decreased by $<50 \%$.

\subsection{Statistical analysis}

Stata SE 12.0 was used for data analysis. Efficiency and side effects are expressed as percentages. The $\chi^{2}$ test was used to compare changes in EEG and MRI findings. A paired t-test was used to detect AED changes. Logistic regression in a multivariable model was used to assess the association between efficiency and potential risk factors. We considered $p$-values of $<0.05$ to be statistically significant.

\section{Results}

\subsection{Patient data}

We enrolled 92 pediatric patients ( 55 males; 37 females) aged 3 months to 10 years $(29.26 \pm$ 24.18 months) (Fig. 1). Patients were on the KD for 1-12 months (mean KD duration 6.68 months). The average duration of patients' epilepsy and treatment with anticonvulsant medication ranged from 1 to 120 (interquartile range [IQR] 4.5, 12,24) months prior to KD commencement.

Twenty-three patients were not included the study. Eight opted to undergo epilepsy surgery or other treatment without ketone production after a routine KD examination, 6 discontinued within 3 days due to food refusal, 5 discontinued due to allergy, and 4 withdrew from ketone production within 1 week at their parents' request. The 92 patients included in this study experienced 1-5 $(2.37 \pm 1.23)$ types of seizures before their treatment with KD therapy: $60.87 \%$ experienced spasms, $44.57 \%$ partial, $40.22 \%$ generalized tonic-clonic, $36.96 \%$ atonic, $35.87 \%$ clonic, $31.52 \%$ tonic, $26.09 \%$ absence seizures, and $8.70 \%$ myoclonic seizures. Seizure etiology was determined in $30.43 \%$ of patients. In this group, $23.91 \%$ of patients were found to have a known genetic etiology, while $6.52 \%$ of patients had a structural abnormality visible on MRI. Of all of the seizures, $69.57 \%$ were of unknown etiology. The SCN1A gene mutation was observed in nine patients, and CDKL5 in two. Other patients had gene mutations such as in GRIN2A, STXBP1, ALG1, $P D H X, P O L G, G A B R D, S T A M B P, S L C 6 A 1$, and chrM-10158. The structural etiology was sequelae from 
intracranial hemorrhage or encephalitis. All children began KD therapy with a 2:1 ratio of fat and non-fat foods. Some patients adjusted the ratio, as allowed in the study protocol, while others (83.70\%) adhered to the 2:1 KD ratio throughout the study period. Blood ketone levels ranged from 2 to $5 \mathrm{mmol} / \mathrm{L}$. Prior to starting KD therapy, all patients had been treated with 1-10 (4.28 \pm 0.19$)$ types of AEDs (Table 1): $75 \%$ received valproate; $70.65 \%$, topiramate; $70.65 \%$, levetiracetam; $66.30 \%$, glucocorticoids; and $55.43 \%$, nitrazepam. If KD therapy was effective, the AED doses were gradually tapered one by one.

Table 1. Baseline characteristics 


\begin{tabular}{|c|c|}
\hline Characteristics & Values \\
\hline Age at KD commencement (months) & $3-110(29.26 \pm 24.18)$ \\
\hline Disease duration (months) & $1-120, \operatorname{IQR}(4.5,12,24)$ \\
\hline $\operatorname{Sex}(M / F)$ & $92(55 / 37)$ \\
\hline Classification of seizure types & $\mathrm{n}(\%)$ \\
\hline Generalized onset & $42(45.65)$ \\
\hline Focal onset & $41(44.57)$ \\
\hline Unknown onset & $9(9.78)$ \\
\hline Etiology & $\mathrm{n}(\%)$ \\
\hline Genetic & $22(23.91)$ \\
\hline Structural & $6(6.52)$ \\
\hline Unknown & $64(69.57)$ \\
\hline $\mathrm{KD}$ ratio & n (\%) \\
\hline $1 / 1$ & $1(1.09)$ \\
\hline $2 / 1$ & $77(83.70)$ \\
\hline $3 / 1$ & $3(3.26)$ \\
\hline $4 / 1$ & $9(9.78)$ \\
\hline $4.5 / 1$ & $2(2.17)$ \\
\hline AEDs & n (\%) \\
\hline Valproate & $69(75.00)$ \\
\hline Topiramate & 65 (70.65) \\
\hline Levetiracetam & $65(70.65)$ \\
\hline Glucocorticoid & $61(66.30)$ \\
\hline Nitrazepam & $51(55.43)$ \\
\hline Oxcarbazepine & $23(25.00)$ \\
\hline Clonazepam & $20(21.74)$ \\
\hline Vigabatrin & $12(13.04)$ \\
\hline
\end{tabular}




$\begin{array}{ll}\text { Lamotrigine } & 12(13.04) \\ \text { Phenobarbital } & 11(11.96) \\ \text { Carbamazepine } & 5(5.43) \\ \text { Zonisamide } & 4(4.35) \\ \text { Clobazam } & 4(4.35) \\ \text { Intravenous immunoglobulin } & 2(2.17) \\ \text { Rapamycin } & 1(1.09)\end{array}$

A total of $92,68,55$, and 29 patients received KD treatment for $1,3,6$, and 12 months, respectively. None exhibited an increase in seizure frequency. At 1, 3, 6, and 12 months, 72/92 (78.26\%), 51/68 (75\%), 44/55 $(80 \%)$, and $27 / 29(93.10 \%)$ of the patients were responders, respectively. Five patients who were not responsive at 3 months were responsive by 6 months (Table 2).

Table 2. KD Efficiency

\begin{tabular}{lcccc}
\hline \multicolumn{1}{c}{ Efficacy (n [\%]) } & 1 month & 3 months & 6 months & 12 months \\
\hline $\mathrm{n}$ & 92 & 68 & 55 & 29 \\
Seizure-free & $10(10.87)$ & $10(14.71)$ & $8(14.55)$ & $9(31.03)$ \\
Improvement ( $\geq 50 \%$ reduction) & $62(67.39)$ & $41(60.29)$ & $36(65.46)$ & $18(62.07)$ \\
No improvement (<50\% reduction) $20(21.74)$ & $17(25.00)$ & $11(20.00)$ & $2(6.90)$ \\
\hline
\end{tabular}

A total of 39 patients (42.39\%) discontinued the diet for various reasons, such as lack of efficacy (71.79\%), caregiver non-compliance (10.26\%), child's refusal to eat $(7.69 \%)$, death $(7.69 \%)$, or side effects (2.56\%) (Table 3). There were three cases of death: one due to sudden invasive Streptococcus pneumoniae infection and empyema during the 10th month of the KD, another due to sudden arrhythmia in the $1 \mathrm{st}$ month of the KD, without any convulsions on the day of death, and the third due to dehydration from diarrhea and vomiting, because he could not receive intravenous rehydration for several days, as his home was far from our hospital; this occurred during the 6th month of the KD.

Table 3. Reasons for discontinuing KD therapy 


\begin{tabular}{ll}
\hline Reasons & n (\%) \\
\hline Lack of efficacy & $28 / 39(71.79)$ \\
Caregiver's non-compliance & $4 / 39(10.26)$ \\
Child's refusal to eat & $3 / 39(7.69)$ \\
Death & $3 / 39(7.69)$ \\
Side effects & $1 / 39(2.56)$ \\
\hline
\end{tabular}

Of the 92 patients, 81 reduced the number of AEDs (12 patients discontinued all AEDs), while 11 underwent no change in AEDs. Among the 81 patients who reduced their AEDs, 11 later increased their AEDs because their convulsions recurred after 6-12 months. All 92 patients had been treated with 1-10 (4.28 \pm 0.19$)$ types of AEDs before KD therapy. At the final follow-up, the mean number of AEDs was $0-4$ $(2.45 \pm 0.17)$. The overall mean reduction in AEDs was $1.83 \pm 0.14(p<0.001)$. The EEG and MRI findings also underwent significant changes $(p<0.001, p=0.04)$ (Table 4).

Table 4. Change in EEG and MRI findings

\begin{tabular}{ccccccccc}
\hline & \multicolumn{2}{c}{ Initial } & & \multicolumn{2}{c}{ Reviewed } & \multirow{2}{*}{$\chi^{2}$} & $\mathrm{p}$ \\
\cline { 2 - 3 } & Normal & Abnormal & & Normal & Abnormal nnncy & & \\
\hline EEG & 2 & 90 & & 15 & 62 & 13.88 & $<0.001$ \\
MRI & 29 & 63 & & 34 & 36 & 4.86 & 0.04 \\
\hline
\end{tabular}

EEG reports were available for all patients. Spike counts were used to evaluate EEGs. Before the KD, the EEG was normal in only 2 of 92 patients; $97.83 \%$ had abnormal EEGs, indicating that $90 / 92(97.83 \%)$ had epileptiform discharges and 58/92 (63.04\%) had slowing waves. Those included 37 patients with hypsarrhythmia, with generalized slowing in 21 of them. After KD therapy for 3-6 months, the EEGs of $77 / 92$ (83.70\%) of the patients were retested; $15 / 77$ (19.48\%) had become normal. Epileptiform discharges ceased in 23/77 (29.87\%) after ketogenesis, decreased in 44/77 (57.14\%), and did not improve in 10/77 (12.99\%) patients. The background of EEGs became normal in 45/77 (58.44\%) after ketogenesis, improved in 22/77 (28.57\%), and exhibited no improvement in 10/77 (12.99\%) after ketogenesis.

MRI reports were available for all patients. Before the KD, the findings were abnormal in 63/92 (68.48\%) patients and normal in 29/92 (31.52\%). After KD therapy for 3-6 months, 70/92 (76.09\%) patients underwent further MRI; abnormal findings were observed in 36/70 (51.43\%) and normal findings were observed in $34 / 70$ (48.57\%) patients; further, $12 / 70$ (17.14\%) exhibited a worsened condition. Among these 12 patients, 6 changed from normal to abnormal. Further, 10 of these 12 had invalid KDs, and 2 underwent baseline MRI 6 months before ketogenesis, during which the seizures were frequent, up to 
dozens of times per day, suggesting that seizures might aggravate MRI. Wide extra-cerebral space, encephalatrophy, and encephalomalacia were the main imaging findings. The patients who responded effectively to the KD exhibited no aggravation due to MRI. With the exception of one patient with glucose transfer factor 1 deficiency, MRI revealed improvement in encephalatrophy after treatment with KD; the other patients exhibited no significant improvement in MRI findings.

Age $(p=0.45)$, duration of disease $(p=0.82)$, type of seizure $(p=0.25)$, etiology $(p=0.30)$, type of AED ( $p$ $=0.85)$, and KD ratio $(p=0.41)$ were not associated with treatment efficacy (Table 5$)$.

Table 5. Factors associated with KD efficacy Hypothesis

\begin{tabular}{lllll}
\hline Factor & OR & $95 \% \mathrm{CI}$ & $\mathbf{z}$ & $\mathbf{p}$ \\
\hline Age & 1.02 & $0.98-1.06$ & 0.76 & 0.45 \\
Disease course & 0.99 & $0.95-1.04$ & -0.230 .82 \\
\hline Seizure type & 1.38 & $0.80-2.38$ & 1.15 & 0.25 \\
\hline Etiology & 1.89 & $0.57-6.26$ & 1.04 & 0.30 \\
\hline AED type & 0.85 & $0.62-1.17$ & -0.990 .32 \\
\hline KD ratio & 0.70 & $0.30-1.64$ & -0.820 .41 \\
\hline
\end{tabular}

Most parents in our study (78.26\%) reported that their children had demonstrated progress in the intellectual, language, or movement components, but the increases of intelligence assessment were not significant $(p>0.05)$. However, $11.96 \%$ reported that their child did not exhibit changes in any of these measures of intelligence, and $9.78 \%$ of parents reported that they could not evaluate their child's progress. Some patients were unable to complete the intelligence assessments due to disturbances in consciousness or their parents' refusal to perform the evaluation; $55.43 \%$ of all patients completed the intelligence assessment and $35.29 \%(18 / 51)$ of the parents reviewed the assessment.

Complications of the KD included transient hyperlipidemia in $55.43 \%$ of patients, hyperuricemia in $2.17 \%$, nephrolithiasis in $2.17 \%$, and hypoalbuminemia in $1.09 \%$. Transient hyperlipidemia and hyperuricemia were reduced to normal without any treatment. Nephrolithiasis disappeared after potassium citrate usage was increased. Hypoalbuminemia was cured by reducing the KD ratio (increasing the protein content).

\section{Discussion}

As the incidence of refractory epilepsy has not decreased despite the emergence of new AEDs, the use of $\mathrm{KD}$ therapy for the treatment of epilepsy has a long history and has attracted more attention in recent years. In our study, $83.70 \%$ patients followed a $2: 1 \mathrm{KD}$ ratio, and $78.26 \%$ responded to the KD by the first 
month of treatment. Our findings are consistent with those of some previous studies ${ }^{[10-13]}$ but different from others ${ }^{[14,15]}$. Our study included a greater number of children who exhibited improvement and whose seizures were managed while following the 2:1 KD ratio.

Five patients in our study who were not responsive to KD therapy at the 3-month evaluation were responsive at 6 months. The treatment was ineffective in all five patients at 3 months, and the doctors recommended stopping KD therapy according to the guidelines. Since no other treatment measures were available, and since the parents had found that the KD had no harmful effects, they insisted on continuing until the 6th month, by which time the KD elicited positive results. Our findings are different from those of $\mathrm{Wu}^{[10]}$, who reported that the efficacy rate did not increase after 3 months.

We also observed a lower rate of side effects than that reported in previous studies, which was as high as $80 \%$ in the study by Lin et al. ${ }^{[16]}$. This may be due to our exclusion of fasting at the beginning of the KD, regular follow-up, or the close contact between doctors and parents (who could contact the doctor directly at any time). The lower incidence as lower ratio often correlates with reduced acidosis.

The patients in our study were followed-up for only 12 months. However, as suggested by some studies, despite being treated with the KD for 1 or 2 years, epilepsy relapses can reoccur in individuals with certain syndromes after withdrawing from KD therapy ${ }^{[17]}$. Therefore, to provide additional information regarding the KD, a longer follow-up is required.

For some epilepsy syndromes such as Dravet syndrome, KD therapy has been reported to be more beneficial than the average KD response rate ${ }^{[17,18]}$. Given the issue of compliance and limited resources, indications for the use of KD treatment should be determined more precisely ${ }^{[19]}$. In our study, the most common reason for discontinuing KD was lack of efficacy (71.79\%). Determining which patients will respond to the diet can help to increase the efficiency of the treatment. Compliance of caregivers with the diet therapy is likely to be higher in patients who respond. Thus, to determine the best candidates for treatment with KD, we analyzed patient characteristics such as age, duration of the disease, type of seizure, etiology, type of AED, and the KD ratio. Regrettably, we found no statistical correlations between those characteristics and the response to the KD. Previous studies ${ }^{[10,20]}$ reported the same finding. This suggests that KD treatment should not be restricted by the above factors. One interesting finding of our study was that one patient discontinued KD for 3 months, and her relapsed convulsions were completely controlled by added topiramate. Another patient's relapse convulsions, after discontinuing all AEDs for 18 months, were controlled with only a small dose of topiramate $(0.5 \mathrm{mg} / \mathrm{kg} / \mathrm{d})$; adding the $\mathrm{KD}$ allowed her convulsions to become controlled. It is important to note that topiramate was not effective before the patient began following the KD. Further studies are needed to determine whether there are synergistic effects between KD therapy and topiramate administration.

$\mathrm{KD}$ can also improve communication in patients with epilepsy and autism spectrum disorders ${ }^{[21,22]}$. The mechanisms associated with the KD are complicated and warrant further study ${ }^{[23]}$. KD has a positive impact on behavioral and cognitive function ${ }^{[24]}$, particularly regarding mood. In our study, most of the 
parents reported that their child exhibited progress on the intellectual, language, or movement components of the intelligence assessment, but the increases were not significant. These results suggest that, by modifying a metabolic mechanism and affecting neuronal excitability, KD not only reduced seizures, but also suppressed the reduction in cognitive function, which is induced by epilepsy. However, the participants' age, cognitive abilities, etiologies, and syndromes varied widely. No standard objective assessment scale was appropriate for the cohort in the present study; therefore, other assessment methods were used to evaluate cognition. The scale used to conduct an objective assessment of cognition in this study requires further standardization and improvement.

The use of a FreeStyle monitoring device for the frequent assessment of serum ketones (and glucose) allows for home monitoring while reducing the need for hospital visits. This test is convenient and rapid; it requires only trace amounts $(1.5 \mu \mathrm{L})$ of blood from the tip of the finger, and beta-hydroxybutyrate can be quickly (within $10 \mathrm{~s}$ ) tested. The test reduces pain in children and limits the need for nurses to collect

blood intravenously. Walta et al. ${ }^{[25]}$ and Chong and Reineke ${ }^{[26]}$ reported that when the blood ketone value is $>4$ or $6 \mathrm{mmol} / \mathrm{L}$, the detected value of the FreeStyle instrument will be lower than the actual value. Currently, all FreeStyle instruments used to detect blood ketones on the market are designed for diabetic patients, and lack specific blood ketone assayers for KD patients. With the use of the KD becoming more common, the research and production of a KD monitoring instrument for high blood ketones is warranted. The use of the FreeStyle instrument for the monitoring of serum ketones is convenient, but there are currently no specific blood ketone assayers for KD patients. The research and production of a KD monitoring instrument for high blood ketones should follow.

The validity of our study is based on the seizure records provided by the parents, which may vary by the carefulness of the observation, which is a defect in this study, but the validity is confirmed by EEG. Our electro-clinical remission rate was higher than that reported by Dressler ${ }^{[27]}$. Most of the patients' EEGs improved in terms of epileptiform discharges. KD failed to treat patients whose MRI findings indicated worsening of the condition. Patients with an effective KD response exhibited no aggravation, suggesting that seizures might aggravate MRI findings.

Genetic testing is still in the developing stages, and some theories of the roles particular genes play remain controversial. For example, we detected pure and mutant TBCD in one patient; since the c. ${ }^{\star} 112$ (exon39) c > T was in a non-coding region, and the software predicted it was benign, the gene was considered nonpathogenic. Because our genetic research conditions are limited, we cannot complete the verification of every suspected epilepsy gene; this is the direction we need to work towards.

\section{Conclusion}

The KD is an effective and well-tolerated therapy for the non-pharmacological treatment of multidrugresistant epilepsy. Five patients who were not responsive to the KD at the 3-month follow-up were eventually responsive at the 6-month evaluation; therefore, we emphasize that KD therapy should be continued for at least 6 months to verify the responders, particularly for patients who have not been

Page $11 / 15$ 
responsive to suitable AEDs. The main reason in this study for discontinuing the KD was lack of efficacy. Samples with different types of seizures should be analyzed in future studies to increase our knowledge of which patients and which syndromes benefit from a KD. Larger samples should be used to evaluate the effects of the KD on intelligence. Genetic information in relation to diet response and the synergistic effect between KD and AEDs is a topic that needs further study.

\section{Abbreviations}

KD: ketogenic diet AED: anti-epileptic drug

EEG: electroencephalography

MRI: magnetic resonance imaging

\section{Declarations}

Ethics approval and consent to participate $\ T$ The study protocol was approved by the Institutional Review Board of the Children's Hospital of Chongqing Medical University. The participants' parents were informed of the risks/benefits of study participation and provided written consent for participation in the present study.

Consent for publication $₫$ Not Applicable.

Availability of data and materials囚http://www.medresman.org.cn/uc/index.aspx

Code availability (software application or custom code): ziyan030815, wangjuan030815

Competing interests: Ms. Juan Wang, Dr. Li Jiang, Dr. Yi Guo and Dr. Min Cheng report no disclosures.

Funding $\triangle$ This work was supported by the National Natural Science Foundation of China (No. 81701277).

Authors' contributions $₫$ All listed authors meet authorship criteria. The specific contributions made by each author are described.

JW and LJ performed the experiments and analyzed the data, JW and YG designed the study, JW and MC wrote the manuscript. All authors discussed the results and commented on the manuscript.

Acknowledgements $₫$ Department of Neurology (or Pediatric Research Institute); Ministry of Education Key Laboratory of Child Development and Disorders; National Clinical Research Center for Child Health and Disorders (Chongqing); China International Science and Technology Cooperation base of Child development and Critical Disorders; Children's Hospital of Chongqing Medical University, Chongqing, P.R China. 
Chongqing Key Laboratory of Translational Medical Research in Cognitive Development and Learning and Memory Disorders

\section{References}

1. Chen Z, Brodie MJ, Liew D, Kwan P. Treatment Outcomes in Patients With Newly Diagnosed Epilepsy Treated With Established and New Antiepileptic Drugs: A 30-Year Longitudinal Cohort Study. JAMA Neurol. 2018; 75:279-86. doi: 10.1001/jamaneurol.2017.3949.

2. Rong P, Liu A, Zhang J, Wang Y, Yang A, Li L, et al. An alternative therapy for drug-resistant epilepsy: transcutaneous auricular vagus nerve stimulation. Chin Med J (Engl). 2014; 127:300-4. PMID: 24438620

3. Wang WZ, Wu JZ, Wang DS, Dai XY, Yang B, Wang TP, et al. The prevalence and treatment gap in epilepsy in China: an ILAE/IBE/WHO study. Neurology. 2003; 60:1544-5. doi: 1212/01.wnl.0000059867.35547.de

4. Chung AM, Eiland LS. Use of second-generation antiepileptic drugs in the pediatric population. Paediatr Drugs. 2008; 10:217-54. doi:2165/00148581-200810040-00003

5. Northam RS, Hernandez AW, Litzinger MJ, Minecan DN, Glauser TA, Mangat S, et al. Oxcarbazepine in infants and young children with partial seizures. Pediatr Neurol. 2005; 33:337-44. doi区 1016/j.pediatrneurol.2005.05.011

6. van der Louw E, van den Hurk D, Neal E, Leiendecker B, Fitzsimmon G, Dority L, et al. Ketogenic diet guidelines for infants with refractory epilepsy. Eur J Paediatr Neurol. 2016; 20:798-809. doi :1016/j.ejpn.2016.07.009

7. Neal EG, Chaffe H, Schwartz RH, Lawson MS, Edwards N, Fitzsimmons G, et al. The ketogenic diet for the treatment of childhood epilepsy: a randomised controlled trial. Lancet Neurol. 2008; 7:500-6. doi: 1016/S1474-4422(08)70092-9

8. Martin-McGill KJ, Jackson CF, Bresnahan R, Levy RG, Cooper Ketogenic diets for drugresistant epilepsy. Cochrane Database of Systematic Reviews. 2018. 7;11(11):CD001903. Doi: 10.1002/14651858.CD001903.pub4

9. Berg AT, Berkovic SF, Brodie MJ, et al. Revised terminology and concepts for organization of seizures and epilepsies: report of the ILAE Commission on Classification and Terminology, 2005-2009. Epilepsia. 2010 Apr;51(4):676-685. doi: :1111/j.1528-1167.2010.02522.x

10. Wu YJ, Zhang LM, Chai YM, Wang J, Yu LF, Li WH, et al. Six-month efficacy of the Ketogenic diet is predicted after 3 months and is unrelated to clinical variables. Epilepsy Behav. 2016; 55:165-9. doi: 1016/j.yebeh.2015.12.008

11. Stenger E, Schaeffer M, Cances C, Motte J, Auvin S, Ville D, et al. Efficacy of a ketogenic diet in resistant myoclono-astatic epilepsy: A French multicenter retrospective study. Epilepsy Res. 2017; 131:64-9. :doi: 1016/j.eplepsyres.2017.02.005 
12. Guzel O, Uysal U, Arslan N. Efficacy and tolerability of olive oil-based ketogenic diet in children with drug-resistant epilepsy: A single center experience from Turkey. Eur J Paediatr Neurol. 2019; 23:14351. :doi: 1016/j.ejpn.2018.11.007

13. Ashrafi MR, Hosseini SA, Zamani GR, Mohammadi M, Tavassoli A, Badv RS, et al. The efficacy of the ketogenic diet in infants and young children with refractory epilepsies using a formula-based powder. Acta Neurol Belg. 2017; 117:175-82. Doi: :1007/s13760-016-0732-0

14. Sampaio L, Takakura C, Manreza M. The use of a formula-based ketogenic diet in children with refractory epilepsy. Arq Neuropsiquiatr. 2017; 75:234-7. Doi: 1590/0004-282X20170028

15. Wijnen B, de Kinderen R, Lambrechts D, Postulart D, Aldenkamp AP, Majoie M, et al. Long-term clinical outcomes and economic evaluation of the ketogenic diet versus care as usual in children and adolescents with intractable epilepsy. Epilepsy Res. 2017; 132:91-9. Doi:

1016/j.eplepsyres.2017.03.002

16. Lin A, Turner Z, Doerrer SC, Stanfield A, Kossoff EH. Complications During Ketogenic Diet Initiation: Prevalence, Treatment, and Influence on Seizure Outcomes. Pediatric Neurol. 2017; 68:35-9. Doi: 1016/j.pediatrneurol.2017.01.007

17. Dhamija R, Eckert S, Wirrell E. Ketogenic diet. Can J Neurol Sci. 2013; 40:158-67. doi: 1017/s0317167100013676

18. Kossoff EH, Zupec-Kania BA, Auvin S, Ballaban-Gil KR, Christina Bergqvist AG, Blackford R, et al. Optimal clinical management of children receiving dietary therapies for epilepsy: Updated recommendations of the International Ketogenic Diet Study Group. Epilepsia Open. 2018; 3:175-92. Doi: 1002/epi4.12225

19. Kossoff EH, Al-Macki N, Cervenka MC, Kim HD, Liao J, Megaw K, et al. What are the minimum requirements for ketogenic diet services in resource-limited regions? Recommendations from the International League Against Epilepsy Task Force for Dietary Therapy. Epilepsia. 2015; 56:1337-42. doi: 1111/epi.13039

20. Kossoff EH, Wang HS. Dietary therapies for epilepsy. Biomed J. 2013; 36:2-8. doi: 4103/23194170.107152

21. Ruskin DN, Svedova J, Cote JL, Sandau U, Rho JM, Kawamura M Jr, et al. Ketogenic diet improves core symptoms of autism in BTBR mice. PLoS One. 2013; 8:e65021. doi: 1371/journal.pone.0065021

22. Herbert MR, Buckley JA. Autism and dietary therapy: case report and review of the literature. J Child Neurol. 2013; 28:975-82. doi: 1177/0883073813488668

23. Maalouf M, Rho JM, Mattson MP. The neuroprotective properties of calorie restriction, the ketogenic diet, and ketone bodies. Brain Res Rev. 2009; 59:293-315. doi: 1016/j.brainresrev.2008.09.002

24. IJff DM, Postulart D, Lambrechts D, Majoie M, de Kinderen R, Hendriksen J, et al. Cognitive and behavioral impact of the ketogenic diet in children and adolescents with refractory epilepsy: A randomized controlled trial. Epilepsy Behav. 2016; 60:153-7. doi: 1016/j.yebeh.2016.04.033 
25. Walta AM, Keltanen T, Lindroos K, Sajantila A. The usefulness of point-of-care (POC) tests in screening elevated glucose and ketone body levels postmortem. Forensic Sci Int. 2016; 266:299-303. doi: 1016/j.forsciint.2016.06.003

26. Chong SK, Reineke EL. Point-of-Care Glucose and Ketone Monitoring. Top Companion Anim Med. 2016; 31:18-26. doi: 1053/j.tcam.2016.05.005

27. Dressler A, Benninger F, Trimmel-Schwahofer P, Gröppel G, Porsche B, Abraham K, et al. Efficacy and tolerability of the ketogenic diet versus high-dose adrenocorticotropic hormone for infantile spasms: A single-center parallel-cohort randomized controlled trial. Epilepsia. 2019; 60:441-51. doi:

1111/epi.14679

\section{Figures}

\begin{tabular}{|c|c|}
\hline 115 patients assessed for eligibility & $\begin{array}{l}8 \text { surgery or other treatment } \\
6 \text { food refusal } \\
5 \text { allergy } \\
4 \text { parents requested to withdraw within } 1 \text { week }\end{array}$ \\
\hline $\begin{array}{c}\text { Baseline } 92 \text { patients started the } \mathrm{KD} \\
\downarrow \downarrow\end{array}$ & \\
\hline $\begin{array}{l}1^{\text {st }} \text { month } 92 \text { patients were on } \mathrm{KD} \\
\qquad \downarrow \rightarrow 24 \text { patients excluded } \rightarrow\end{array}$ & $\begin{array}{l}19 \text { lack of efficacy } \\
2 \text { caregiver non-compliance } \\
2 \text { food refusal } \\
1 \text { death }\end{array}$ \\
\hline$\downarrow \quad \rightarrow 13$ patients excluded $\rightarrow$ & $\begin{array}{l}4 \text { followed - up for less than } 6 \text { months } \\
5 \text { lack of efficacy } \\
2 \text { caregiver non-compliance } \\
1 \text { food refusal } \\
1 \text { death }\end{array}$ \\
\hline $\begin{aligned} & \downarrow \rightarrow 26 \text { patients excluded } \rightarrow \\
& 12^{\text {th }} \text { month } 29 \text { patients were on } \mathrm{KD}\end{aligned}$ & $\begin{array}{l}20 \text { followed - up for less than } 12 \text { months } \\
4 \text { lack of efficacy } \\
1 \text { death } \\
1 \text { side effects }\end{array}$ \\
\hline
\end{tabular}

\section{Figure 1}

Flow of patients in the study 